\title{
Mining and predictive characterization for resistance to leaf rust (Puccinia hordei Otth) using two subsets of barley genetic resources
}

\section{Mariam Amouzoune}

ICARDA: International Center for Agricultural Research in the Dry Areas

Ahmed Amri

ICARDA: International Center for Agricultural Research in the Dry Areas

\section{Rachid Benkirane}

Ibn Tofail University Kenitra: Universite Ibn Tofail Kenitra

\section{Zakaria Kehel}

ICARDA: International Center for Agricultural Research in the Dry Areas

\section{Muamer Al-Jaboobi}

ICARDA: International Center for Agricultural Research in the Dry Areas

\section{Adil Moulakat}

ICARDA: International Center for Agricultural Research in the Dry Areas

\section{Jilal Abderrazek}

Institut National de Recherche pour l'Agriculture l'Alimentation et l'Environnement

Sajid Rehman ( $\nabla$ srehman@perennia.ca )

Perennia Food and Agriculture Inc.

\section{Research Article}

Keywords: Barley, Puccinia hordei, Resistance, Genetic resources, Efficient mining, Machine learning.

Posted Date: April 16th, 2021

DOI: https://doi.org/10.21203/rs.3.rs-394214/v1

License: (c) (1) This work is licensed under a Creative Commons Attribution 4.0 International License. Read Full License

Version of Record: A version of this preprint was published at Genetic Resources and Crop Evolution on October 2nd, 2021. See the published version at https://doi.org/10.1007/s10722-021-01268-4. 


\section{Mining and predictive characterization for resistance to leaf rust}

\section{resources}

4

5

6

7

8

9

Mariam.amouzoune@gmail.com

Ahmed Amri

a.amri@cgiar.org

Rachid Benkirane

rachid.benkirane@yahoo.fr

Zakaria Kehel

Z.Kehel@cgiar.org

23 Muamer Al-Jaboobi

moumr@hotmail.com

24 Adil Moulakat

A.Moulakat@cgiar.org

25

Jilal Abderrazek

jilal abderrazek_2001@yahoo.fr

Sajid Rehman

srehman@perennia.ca 


\section{Abstract}

28 Sustainable barley production will require access to diverse $e x$-situ conserved collections to develop varieties with 29 high yields and capable of overcoming the challenges imposed by major abiotic and biotic stresses. This study 30 aimed at searching efficient approaches for the identification of new sources of resistance to barley leaf rust (LR). 31 Two subsets, Generation Challenge Program Reference set (GCP) with 190 accessions and leaf rust subset 32 constructed using the filtering approach of the Focused Identification of Germplasm Strategy (FIGS) with 100 accessions, were evaluated for the seedling as well as the adult plant stage resistance (APR) using two LR isolates (ISO-SAT and ISO-MRC) and in four environments in Morocco, respectively. Both subsets yielded a high percent of accessions with a moderately resistant (MR) reaction to the two LR isolates at the seedling stage. For APR, more than 50\% of the accessions showed resistant reactions in SAT2018 and GCH2018, while this rate was less than 20\% in SAT2017 and SAT2019. Statistical analysis using chi-square test of independence revealed the dependency of LR reaction on subsets at the seedling (ISO-MRC), as well as at the APR (SAT2017 and SAT2018) stage. Furthermore, the test of goodness of fit showed that FIGS_LR yielded higher percentages of resistant accessions than GCP subset in case of ISO-MRC at the seedling stage, and in case of SAT2017 and SAT2018 at APR stage. Although some of the tested machine learning models had moderate to high accuracies, none of them was able to find a strong and significant relationship between the reaction to LR and the environmental conditions showing the needs for more fine tuning of approaches for efficient mining of genetic resources using machine 44 learning.

Keywords Barley, Puccinia hordei, Resistance, Genetic resources, Efficient mining, Machine learning. 
Cultivated barley (Hordeum vulgare subsp. vulgare L.) is the fourth most important cereal crop in the world after wheat, maize, and rice, in terms of production of 143.13 million metric tons and acreage of around 47.37 million hectares (FAOSTAT 2017; USDA 2019). In Morocco, barley is grown on 2 million hectares in the arid and semiarid regions with $1.23 \mathrm{t} / \mathrm{ha}$ of average grain yield, which is relatively low compared to North America (3.67 t/h) (FAOSTAT 2017). The lower national average grain yield of barley is due to limited or no use of inputs, and the prevalence of abiotic and biotic constraints. Foliar diseases such as powdery mildew, net blotch, spot blotch, and leaf rust are important biotic constraints that limit the grain and straw yields and their quality. Barley leaf rust caused by Puccinia hordei Otth $(P h)$ is one of the most destructive and globally spread barley diseases (Clifford 1985; Park et al. 2015). It is widely distributed throughout barley growing area, and can cause serious yield losses in the regions of North Africa, Europe, New Zealand, Australia, the Eastern and Midwestern parts of United States, and some parts of Asia, where susceptible and late maturity varieties of barley are sown (Arnst et al. 1979; Clifford 1985; Chicaiza et al. 1996; Brunner et al. 2000, Niks et al. 2000). Losses of barley production due to LR can reach up to $30 \%$ on susceptible cultivars (Cotterill et al. 1992, Griffey et al. 1994).

Applying fungicides is an efficient strategy to control major foliar diseases, but it is not economical for barley grown under marginal lands and low-input conditions of Morocco. Therefore, the use of resistant varieties is the most effective, economical, and environmentally safe way for controlling barley LR. This can be achieved by transferring identified resistant genes from diverse genetic resources into elite barley germplasm (Hajjar and Hodgkin, 2007; Rehman et al. 2020). To date, 23 Rph genes conferring hypersensitive resistance to barley leaf rust at the seedling stage (Rph1 to Rph19, Rph21, and Rph22, Rph25, Rph26), and 3 APR genes (Rph20, Rph23, Rph24) have been identified from Hordeum vulgare subsp. vulgare, or transferred from H. vulgare subsp. spontaneum, and H. bulbosum (Park 2015; Kavanagh et al. 2017; Yu et al. 2018). However, LR resistance faces a big challenge from a rapidly evolving pathogen due to recombination and mutations which leads to the development of new pathotypes that overcome deployed single major Rph genes in a short time span (McIntosh 1988; Figueroa et al. 2016). Most of the barley varieties released in Morocco are susceptible to LR, and limited sources of resistance are available against the LR populations prevailing in the northern parts of Morocco. Therefore, it is required to evaluate and identify continuously new sources of resistance from existing germplasm, and from gene bank collections (Qualset 1975; Sing et al. 2015).

The genetic resources remain the most important source of parental germplasm for barley breeding programs to develop new varieties with high yield, better end-use quality, tolerant to abiotic stresses, and resistant to major diseases and pests. But the search for a given trait is limited owing to the large number of accessions being held in the genebanks. Further, the evaluation of these large collections for some traits can be very expensive. To facilitate the screening and the mining of genetic resources, it requires the development of intelligent subsetting approaches to fit the available funding and facilities (ICARDA 2015). These approaches aim to select subsets from the original collection to harness maximum diversity within limited number of accessions (Gollin et al. 2000). Frankel and Brown (1984) recommended the use of core collection which selects 5-10\% of the original collection, representing maximum geographical or morphological diversities. However, because of the large number of accessions in the entire collection in genebanks, even a core collection can still be unmanageable for the evaluation of some traits, and other sub-setting approaches were suggested. Mini-core collections were 
suggested by Upadhyaya and Ortiz (2001) to concentrate broad genetic diversity in smaller subsets. It allows selecting about $1 \%$ of the total accessions from the entire collection and the core collection to represent maximum diversity. The Generation Challenge Program (GCP) (https://www.generationcp.org) recommended the development of a reference set representing $10 \%$ of the core collection to represent maximum diversity using molecular markers.

From ICARDA barley in-trust collection totaling more than 32,000 accessions, the barley core collection (composite set) of 3,000 accessions of both cultivated and wild progenitor species (H. spontaneum) was selected based on climatological data of the collection sites; from which the Generation Challenge Program (GCP) developed a reference subset of 300 accessions based on the diversity of EST-derived, and genomic SSR markers (https://www.croptrust.org/wp/wp-content/uploads/2014/12/Barley_Strategy FINAL 27Oct08.pdf). Though, many researchers have reported on the limitations of core collections in capturing rare and adaptive alleles (Dwivedi et al. 2008; Xu 2010).

The Focused Identification of Germplasm Strategy (FIGS) was developed by ICARDA in collaboration with the Australian and the Russian partners as an alternative approach for efficient mining of genetic resources that maximize the likelihood of capturing specific adaptive traits in subsets of manageable size extracted from the original collection (Mackay 1990; Street et al. 2008). FIGS is based on the co-evolution between the accessions and the environmental conditions in which they evolved (Mackay 1995; Gollin et al. 2000; Mackay and Street 2004; Bari et al. 2012). This approach exploits the development of the relationship between the specific soughttrait and ecogeographical data by filtering germplasm collections through exerting selection pressures of the emergence of a sought trait. It uses also the modeling pathways. When the relationship is confirmed, a manageable subset can be selected to include accessions with high probability of having the sought trait. FIGS subsets have allowed to identify for the first-time sources of resistance to Sunn pest in wheat (El Bouhssini et al. 2009), resistance to net blotch in barley (Endersen et al. 2011), and drought tolerance in faba bean (Khazaei et al. 2014). The present study aimed at: i) Identification of sources of resistance to LR in FIGS_LR and GCP subsets; ii) Assessing the dependence of resistance on sub-setting approach; and iii) search for the best model that describes the relationship between resistance to LR, and the environmental conditions using machine learning.

\section{Materials and Methods}

Plant material

Two barley subsets extracted from ICARDA in-trust collection available from the regeneration efforts conducted in Morocco to reconstruct the active and base collections were used in this study. A total of 188 accessions from the reference set constructed within the Generation Challenge Program (GCP) and extracted from the composite set of barley collection held at ICARDA based on diversity of EST-derived and genomic SSR markers (Supplementary Table S1). Another subset composed of 86 accessions was selected using filtering approach of the Focused Identification of Germplasm Strategy (FIGS_LR) based on the following parameters:

$\checkmark$ Count number of days where the average daily temperature is between $8-15^{\circ} \mathrm{C}, 10$ days before the onset of growing period and up to $15 \%$ into the vegetative phase.

$\checkmark \quad$ Remove sites with zero count from step 1 . 
$\checkmark$ Sum daily rain for 10 days before the onset of growing period up to $10 \%$ into the vegetative phase.

$\checkmark$ Normalize both variables (steps 1 and 2) to range $0-1$ for each site.

$\checkmark \quad$ Add variables to create index 1 .

$\checkmark$ Rank based on index 1 and remove bottom 25 percent of sites.

For the remaining sites, the following was done:

$\checkmark$ From $10 \%$ into the vegetative phase until onset of grain filling divide into 3 separate sub-phases of equal length.

$\checkmark$ For each sub-phase count the number of days where the average daily temperature is between $18-20^{\circ}$

C.

$\checkmark \quad$ For each sub-phase determine the amount of precipitation.

$\checkmark$ Remove sites if any of the variables $=0$ (3 count variables and 3 precipitation variables).

$\checkmark$ Normalize each variable for a range between $0-1$.

$\checkmark \quad$ Add each variable and then add index 1 to create index 2 .

$\checkmark$ Rank sites using index 2 from largest to smallest.

$\checkmark$ Since there are more sites than the desired set size, then one accession could be chosen randomly from each site starting at the top ranked site until the desired set size is reached. Alternatively, this approach could be taken after one candidate accession is donated by each country represented in the candidate site list.

The climatic conditions layers were extracted from the GIS surfaces modeled from data collection sites as described by De Pauw (2008). The FIGS-LR subset has more accessions from Greece, Turkey, Ethiopia, and India (Supplementary Table S2).

\section{Seedling screening}

The seedling screening of GCP and FIGS_LR subsets was conducted under controlled conditions in the growth chamber with two pure isolates of LR (ISO-SAT and ISO-MRC). The single urediniospore was isolated from infected leaves collected from the experimental stations of Sidi Allal Tazi (ISO-SAT) and Marchouch (ISO-MRC) in 2017 and were multiplied on the susceptible barley cultivars (Bowman and Aglou) followed by collection and drying of urediniospores on silica gel and storage at $-80{ }^{\circ} \mathrm{C}$ until further use.

Barley plants were grown in sterilized peat moss (supplemented with 14-14-14 NPK) in plastic cones (14 cm long cones with $3.8 \mathrm{~cm}$ diameter) positioned in a $14 \times 7$-unit tray (Steuwe \& Sons, Inc., OR, United States). For each barley accession, 4-5 seeds were planted per cone in two replications. Each tray contained 96-test genotypes along with resistant (Philadelphia) and susceptible (Bowman) checks. Plants were raised in the growth chamber (Snijder Scientific, Tilburg, the Netherlands) with a photoperiod of $16 \mathrm{~h} \mathrm{light} / 8 \mathrm{~h}$ dark at $20 \pm 1^{\circ} \mathrm{C}$. Inoculation was carried out on 10-12 days old seedlings when the first leaf was fully expanded. To prepare LR inoculum, urediospores were taken from the $-80^{\circ} \mathrm{C}$ freezer and subjected to heat shock for 5 min at $40{ }^{\circ} \mathrm{C}$. For each tray, $15 \mathrm{mg}$ of urediniospores were suspended in $10 \mathrm{ml}$ of light mineral oil (Novec 7100, Sigma Aldrich), and this spore suspension was sprayed onto plants as a fine mist using an airbrush (Revell, Munchen, Germany). Inoculated plants were left to dry for 20 minutes at the room temperature and were placed in growth chamber in the dark for 24 hours at $18{ }^{\circ} \mathrm{C}$ with $\sim 100 \%$ relative humidity. Then the plants were maintained in the growth 
chamber with a light/ dark period of $16 / 8 \mathrm{~h}$ at $20^{\circ} \mathrm{C}$ for symptoms development. The evaluation for LR disease was carried out 12-14 days post-inoculation based on infection types (ITs) according to the 0 to 4 scale developed by Stakman et al. (1962). The seedlings were classified either as immune (0), resistant (0; and 1), moderately resistant (2), moderately susceptible (3), or susceptible (4).

Field screening

The field phenotyping was performed at the INRA experimental station of Sidi Allal Tazi $\left(34^{\circ} 52^{\prime} \mathrm{N}, 6.32\right.$ W) during 2016-17 (SAT2017), 2017-18 (SAT2018), 2018-19 (SAT2019), at Marchouch (33 56'10”N 669'21'W) during 2017-18 (MRC2018), and at Guich (3358'59.7"N 651'41.6"W) during 2017-18 (GCH18). The tested accessions were sown as single rows of $1 \mathrm{~m}$ with $0.5 \mathrm{~m}$ row spacing between adjacent accessions using an augmented block design. The seed mixture of susceptible cultivars Bowman and Aglou were sown as a rust spreader row at the border of each block to allow the uniform distribution of LR inoculum.

At SAT, the disease was established naturally, but at Guich station the disease was initiated using the artificial inoculation. About 1 gram of dried urediniospores were suspended in $200 \mathrm{ml}$ of mineral oil and sprayed on the trial using an airbrush (Revell, Munchen, Germany). The establishment and spread of the disease were favored by covering the spreader rows with a plastic sheet overnight and by periodic sprinkler irrigation. The phenotypic evaluation for LR resistance was assessed for GCP and FIGS_LR subsets at growth stage 65-77 (Zadoks et al. 1974) using the modified Cobb scale (Peterson et al. 1948) which combined the LR severity (0 to $100 \%)$ and host response; 0 (Immune), No visible infection on plants; R (resistant), visible chlorosis or necrosis, no uredia are present; MR (moderately resistant), small uredia are present and surrounded by either chlorotic or necrotic areas; MS (moderately susceptible), medium sized uredia are present and possibly surrounded by chlorotic areas; S (susceptible), large uredia are present, generally with little or no chlorosis and no necrosis. The Coefficient of Infection (CI) was calculated by multiplying the infection response values $(\mathrm{R}=0.2, \mathrm{MR}=0.4, \mathrm{MS}=0.8, \mathrm{~S}=1)$ with the percent disease severity (0-100\%) (Stubbs et al.1986), and the accessions were rated based on the average coefficient of infection (ACI) where values of 0-7, 8-16, 17-29, 30-50, and >50 were considered as resistant, moderately resistant, moderately susceptible, susceptible, and highly susceptible, respectively.

Data analysis

Comparing the reactions of GCP and FIGS subsets

The statistical analysis was performed using R software (R Core Team 2018). The statistical association between sub-setting approach and the reaction to LR was calculated using $\chi^{2}$ test of independence with significance level $(\alpha=0.05)$ using the following equation:

$$
\chi^{2}=\sum_{i=1}^{r} \sum_{j=1}^{c} \frac{\left(O_{i j}-E_{i j}\right)^{2}}{E_{i j}}
$$

The equation used for calculating expected values in a test of independence was as follows: 


$$
E_{i j}=\frac{\sum_{k=1}^{c} O_{i j} \sum_{k=1}^{r} O_{k j}}{N}
$$

208

209

210

211

212

213

214

215

Where $E_{i j}=$ expected value, $\sum_{k=1}^{c} O_{i j}$ is the sum of the $\mathrm{i}^{\text {th }}$ column, $\sum_{k=1}^{r} O_{k j}$ is the sum of the $\mathrm{k}^{\text {th }}$ row, $\mathrm{N}$ is the total number.

To find out the differences between FIGS and GCP subsets in terms of reaction to LR, the test of goodness of fit using $\chi^{2}$ test at a significance level $(\alpha=0.05)$ was used where GCP was simulated to a random sample. The expected values for the test of goodness of fit are calculated as follows:

$$
E_{i}=n p_{i}
$$

Where $E_{i}$ is the expected value, $n$ is the total sample size, and $p_{i}$ is the hypothesized proportion of observations in level $i$.

Both tests were performed using different groupings of reactions, all classes (I, R, MR, MS and S), three classes $(\mathrm{I}+\mathrm{R}, \mathrm{MR}+\mathrm{MS}, \mathrm{S})$ and $(\mathrm{I}+\mathrm{R}+\mathrm{MR}, \mathrm{MS}, \mathrm{S})$, two classes $(\mathrm{I}+\mathrm{R}+\mathrm{MR}, \mathrm{MS}+\mathrm{S})$ at the seedling stage, and all classes (R, MR, MS, S, HS), three classes (R, MR+MS, and S+HS), and two classes $(\mathrm{R}+\mathrm{MR}+\mathrm{MS}, \mathrm{S}+\mathrm{HS})$ at the adult plant stage.

Modeling of the reaction to leaf rust disease

The second pathway of the focused identification of the germplasm strategy (FIGS) using machine learning was investigated using the available reactions of the accessions of FIGS_LR and GCP subsets to find a function that links adaptive traits, environments (and associated selection pressures) with genebank accessions. We used environmental data from WorldClim1 databases as predictors. The WorldClim is an open access database providing global climatic layers describing past climatic profiles of collection sites intended for spatial modeling or mapping. It includes averages of monthly minimum and maximum temperatures, precipitation and bioclimatic variables (Fick and Hijmans 2017).

The following machine learning algorithms were used: K-nearest neighbors KNN (Kotsiantis 2007), Support Vector Machine SVM (Hsu et al. 2010), Random Forest RF (Breiman, 2001), Neural networks NNET (Venables and Ripley 2002), and Bagged Carts BCART (Kołcz 2000). Each machine learning model was tuned to select the best tuning parameters using a training set ( $70 \%$ of the total set), and then the best model was selected between different machine learning models based on several metrics including accuracy, specificity, and Kappa. The modeling metrics were computed on the test set (30\% of the total set). In this study, R language and caret library were used for machine learning analysis (Kuhn 2008). Models were tuned for parameter's optimization and trained on $70 \%$ of the data and tested with 10 cross validation folds and 100 replications. In addition, modeling was done for the two isolates for the seedling stage. For the APR, modeling was done for the entire multi-locations data sets and for each location separately. 


\section{Results}

Seedling resistance

In the seedling test, successful artificial inoculation was carried out for the two isolates and diverse infection responses were recorded. The frequency distribution of infection response of GCP and FIGS_LR accessions at the seedling stage has been presented in Figure 1. The uniformity of the disease development was assessed through the high susceptibility of the check Bowman over the two replications. The highest number of accessions of both FIGS_LR (65.88 \% for ISO-MRC, and $51.16 \%$ for ISO-SAT), and GCP (62.87 and $48.4 \%$ for ISO-MRC and ISO-SAT isolates, respectively) subsets showed MR reaction, and only few accessions showed R reaction with one accession of GCP having immune reaction (Figures 1a and 1b). More resistant accessions were noted when tested to ISO-MRC isolate with 5.88 \% for FIGS_LR and $22.75 \%$ for GCP compared with ISO-SAT isolate that showed $10.47 \%$ and $13.3 \%$ resistant accessions for FIGS_LR and GCP, respectively. Some accessions showed different disease reaction for the two isolates. For example, the accessions IG 143872, IG 143862, IG 143864, IG 143872, IG 143890, IG 143978, IG 143984, IG 144006, IG 144090, IG 144029, IG 144077, IG 143871, and IG 144012 were resistant to ISO-MRC isolate but not to ISO-AT isolate, whereas accessions IG 143963, IG 18725, IG 19525 were resistant to ISO-AT isolate but not to ISO-MRC isolate. Ten barley accessions in GCP (IG 143876, IG 143886, IG 143906, IG 143929, IG 143998, IG 143999, IG 144014, IG 144064, IG 144076, IG 144108) and one in FIGS_LR (IG 18957) were resistant to both isolates. Most of the resistant accessions originated from USA, Turkey, Greece, and Morocco.
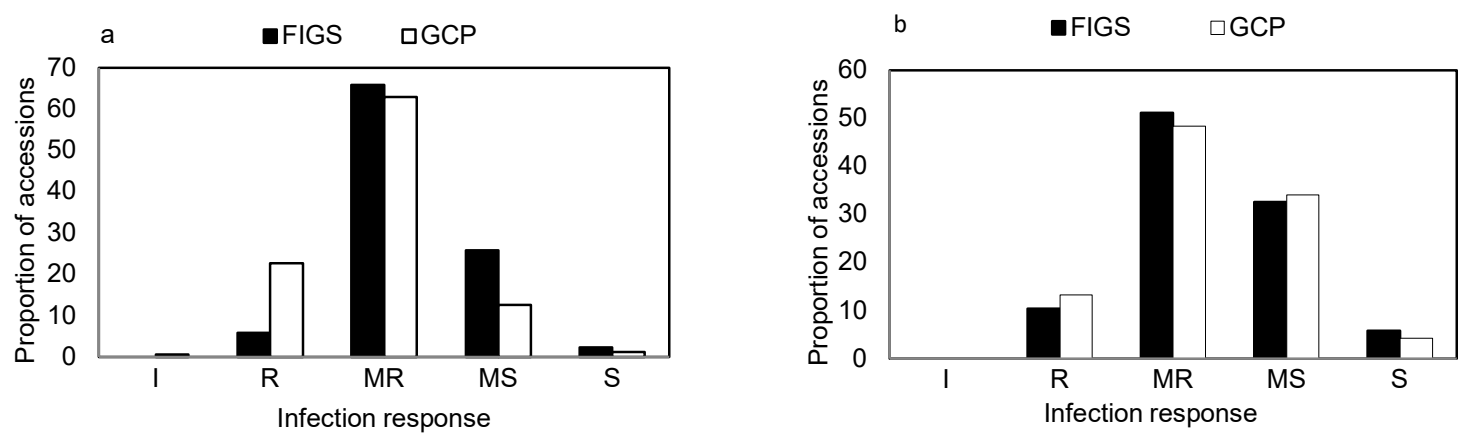

Fig. 1 Frequency distributions of LR disease reactions of barley FIGS_LR and GCP subsets evaluated at the seedling stage against ISO-MRC (a) and ISO-SAT isolates (b). Here I = immune, $\mathrm{R}=$ resistant, MR = moderately resistant, $\mathrm{MS}=$ moderately susceptible, and $\mathrm{S}=$ susceptible.

Adult Plant Resistance (APR)

Under field conditions, good natural LR infection was obtained at the Sidi Allal Tazi during three cropping seasons, and good artificial infection was established at Guich in 2018. However, late and light artificial infection at Marchouch during 2017 and 2018 seasons did not allow disease severity assessments. The uniformity of the disease development was assessed through the high susceptibility of the checks, Bowman and Aglou, at the adult stage in Sidi Allal Tazi and Guich sites. The good development of the disease allowed efficient screening of the germplasm 
at the adult plant stage, as shown by wide range of reactions observed (Figure 2). The average of coefficient of infection $(\mathrm{ACI})$ values across the environments ranged from 0 to 85 with several accessions showing contrasting reactions in different environments. Accessions of FIGS_LR and GCP subsets showed different distributions of the reaction classes with near normal distribution for SAT2017 and SAT2019, and positive skewness with high percentage of R accessions (ranged from 55.8 to 68.3\%) for SAT2018 and GCH2018 (Figures 2b and 2c). While at SAT2017 and SAT2019, this rate ranged from 5.59 to $19.64 \%$ (Figures. 2a and 2d) for both subsets. When considering MR reaction, additional 8.94 to $30.36 \%$ accessions were found during SAT2017 and SAT2019 cropping seasons. The highest numbers of susceptible accessions were observed at SAT2017 with 34.64 and $15.29 \%$ showing susceptibility, and $22.91 \%$ and $15.29 \%$ were highly susceptible (HS) for GCP and FIGS_LR, respectively. Only three accessions in FIGS_LR (IG 28636, IG 28647 and IG 33039), and four accessions in GCP subset (IG 143945, IG 144000, IG 144064, IG 144105) were found to be resistant across all environments (Table $1)$.
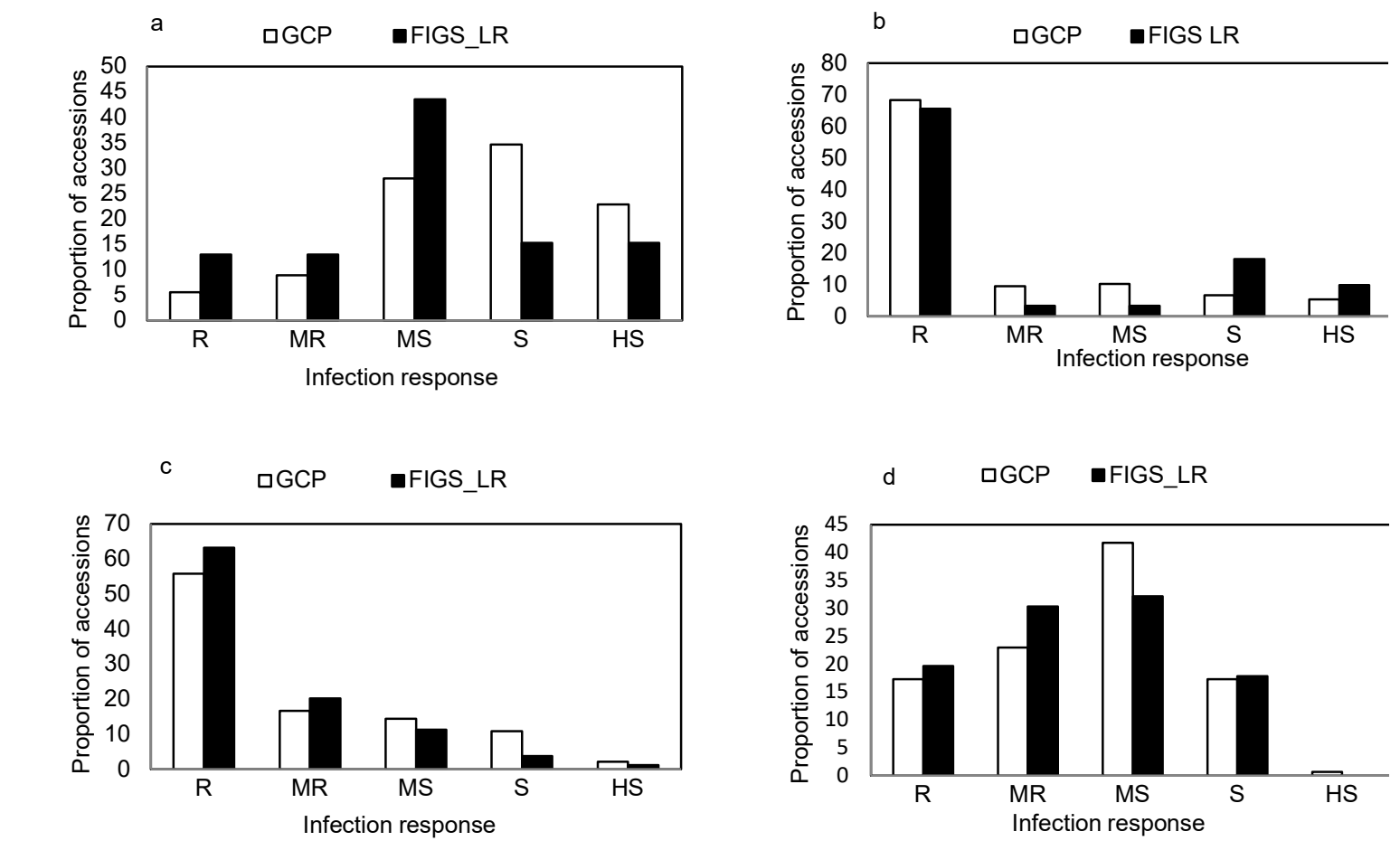

Fig. 2 Frequency distributions of the adult plant resistance (APR) of FIGS_LR and GCP subsets to leaf rust under field conditions at Sidi Allal Tazi during (a) 2017 (SAT2017), (b) 2018 (SAT2018), (d) 2019 (SAT2019) cropping seasons, and at Guich during 2018 (GCH2018 (c)). Here R = resistant, MR= moderately resistant, MS $=$ moderately susceptible, and $\mathrm{S}=$ susceptible. 
Table 1 Average of coefficient of infection (ACI) values of most resistant accessions at the adult plant stage across environments, and their infection responses (IR) at the seedling stage to LR isolates (ISO-MRC, ISOSAT).

\begin{tabular}{llcccccc}
\hline Accession & Subset & SAT2017 & SAT2018 & GCH2018 & SAT2019 & ISO-MRC & ISO-SAT \\
\hline IG 143945 & GCP & 8 & 6.2 & 2.5 & 6 & 2 & 2 \\
IG 144000 & GCP & 12 & 2 & 6.8 & 4 & 2 & 2 \\
IG 144064 & GCP & 2 & 0.8 & 0.4 & 6 & 1 & $0 ;$ \\
IG 144105 & GCP & 4.2 & 0.4 & 1 & 12 & 2 & 3 \\
IG 28636 & FIGS & 6 & 1.1 & 4.95 & 5 & 2 & 2 \\
IG 28647 & FIGS & 6.1 & 4.1 & 0.7 & 8 & 2 & $2+$ \\
IG 33039 & FIGS & 4.2 & 0.4 & 6.7 & 0.2 & 2 & 2 \\
\hline
\end{tabular}

326

327

328

329

330

331

332

333

334

335

336

337

338

339

340

341

342

343

344

345

346

347

348

349

350

351

352

353

354

355

Comparison of the reaction of FIGS_LR and GCP to leaf rust

For ISO-MRC isolate at the seedling stage, GCP subset showed higher percentage of resistant accessions $(22.75$ \%) than FIGS_LR (6\%). Similarly, 13.3\% of GCP accessions and 10.5\% of FIGS_LR accessions were resistant to ISO-SAT isolate. While approximately similar percentages were found for MR and S reaction classes for both isolates (Figures 1a and 1b). At the adult plant stage, FIGS_LR had slightly higher percentages of accessions with $\mathrm{R}$ and MR reactions except at Sidi Allal Tazi during 2017-18 (SAT2018) season (Figure. 1b). Under severe epidemic of LR as observed at Sidi Allal Tazi during 2016-17 season (SAT2017), FIGS_LR showed less accessions having S and HS reactions compared to GCP subset (Figures. 1a and 1c).

Applying the chi-square tests of independence to both subsets, the results showed that there is a significant relationship (P-value=0.04) between the response to LR disease and the sub-setting approach for ISO-MRC isolate, but not for ISO-SAT, when considering all classes of reaction. For ISO-MRC, GCP included one accession with immune reaction and $23 \%$ of the accessions being resistant, while FIGS_LR subset showed only $6 \%$ of the accessions being resistant (Table 2, Figure 1a). When screened with ISO-SAT isolate, both FIGS_LR and GCP showed that the infection response to LR was not dependent on the sub-setting $(\mathrm{p}=0.85)$, displaying same distribution patterns of the reaction. No accession was found immune in both subsets while $9(10.47 \%)$ and 25 (13.3\%) accessions were resistant, and 44 (51.16\%) and 91 (48.4\%) were MR for FIGS_LR and GCP subsets, respectively (Table 2, Figure 1b).

The test of goodness of fit showed that FIGS_LR subset yielded higher percentages of accessions with R, and MR reactions than GCP in case of ISO-MRC isolate, but no significant differences were observed between the two subsets when tested with ISO-SAT isolate. 
Table 2 Number of accessions per reaction type of barley FIGS_LR and GCP subsets evaluated at the seedling stage using two leaf rust isolates (ISO-MRC and ISO-SAT) with $\chi^{2}$ (P-values) for the tests of independence and goodness of fit.

\begin{tabular}{ccccc}
\hline & \multicolumn{2}{c}{ ISO-MRC } & \multicolumn{2}{c}{ ISO-SAT } \\
\cline { 2 - 5 } & FIGS_LR & GCP & FIGS_LR & GCP \\
\hline I & 0 & 1 & 0 & 0 \\
R & 5 & 38 & 9 & 25 \\
MR & 56 & 105 & 44 & 91 \\
MS & 22 & 21 & 28 & 64 \\
S & 2 & 2 & 5 & 8 \\
\hline$* \chi 2(P)$ & $16.31(0.04)$ & \multicolumn{2}{c}{$0.81(0.85)$} \\
\hline$* * \chi 2(P)$ & \multicolumn{2}{c}{$1.2(0.75)$} \\
$* \chi 2$ test of independence, and ** 24 goodness of fit test.
\end{tabular}

359

360 361

362

363

364

365

366

367

368

369

370

371

372

373

374

375

376

377

378

379

380

Here I: immune; R: resistant; MR: moderately resistant; MS: moderately susceptible; S: susceptible.

Except the grouping of two classes (I+R+MR, MS+S) for the goodness of fit test, the tests of independence and goodness of fit were significant for ISO-MRC, but not for ISO-SAT for different groupings of the reactions (Table $3)$.

Table $3 \chi^{2}$ (P-value) for the tests of independence and goodness of fit for different groupings of the reaction to two leaf rust isolates (ISO-MRC and ISO-SAT) at the seedling stage for FIGS-LR and GCP subsets.

\begin{tabular}{lllll}
\hline \multirow{2}{*}{ Groups of reaction } & \multicolumn{2}{l}{ Test of independence } & \multicolumn{2}{l}{ Test of goodness of fit } \\
\cline { 2 - 5 } & ISO-MRC & ISO-SAT & ISO-MRC & ISO-SAT \\
\hline I+R, MR+MS, S & $14.65(0.001)$ & $0.70(0.71)$ & $15.13(0.001)$ & $1.03(0.60)$ \\
I+ R+MR, MS, S & $11.67(0.003)$ & $0.34(0.84)$ & $9.54(0.008)$ & $0.55(0.76)$ \\
I+R+MR, MS+S & $10.10(0.001)$ & $0.0001(0.99)$ & $6.61(0.10)$ & $0.0002(0.99)$ \\
\hline
\end{tabular}

Here I: immune; R: resistant; MR: moderately resistant; MS: moderately susceptible; S: susceptible.

For APR, the reaction to LR was dependent on subsets for Sidi Allal Tazi during 2017 (SAT2017) and 2018 (SAT2018) with respective $\chi^{2}$ (P-values) of 0.001 and 0.02 , respectively. But this dependence was not found in case of GCH 2018 (GCH18) and Sidi Allal Tazi 2019 (SAT2019) (Table 4). The tests of goodness of fit showed that FIGS-LR outperformed GCP subset at Sidi Allal Tazi with higher percentages of accessions with R and MR reactions under heavy infection in 2017, but the opposite was observed during 2018 season at the same site. For GCH2018 and SAT2019 environments, no significant differences were observed between the two subsets. 
Table 4 Number of barley accessions per leaf rust reaction type of FIGS-LR and GCP subsets evaluated at the adult plant stage at Sidi Allal Tazi (SAT) and Guich $(\mathrm{GCH})$ stations with $\chi 2$ (P-values) for the tests of independence and goodness of fit.

\begin{tabular}{ccccccccc} 
& \multicolumn{2}{c}{ SAT2017 } & \multicolumn{2}{c}{ SAT2018 } & \multicolumn{2}{c}{ GCH2018 } & \multicolumn{2}{c}{ SAT2019 } \\
\cline { 2 - 8 } & FIGS_LR & GCP & FIGS_LR & GCP & FIGS_LR & GCP & FIGS_LR & GCP \\
\hline R & 11 & 10 & 40 & 114 & 50 & 77 & 11 & 24 \\
MR & 11 & 16 & 2 & 16 & 16 & 23 & 17 & 32 \\
MS & 37 & 50 & 2 & 17 & 9 & 20 & 18 & 58 \\
S & 13 & 62 & 11 & 11 & 3 & 15 & 10 & 24 \\
HS & 13 & 41 & 6 & 9 & 1 & 3 & 0 & 1 \\
\hline$*{ }^{2}($ P) & $18,3(0,001)$ & $12,26(0,02)$ & $4,46(0,35)$ & & $2,33(0,67)$ \\
\hline$* * \chi 2(P)$ & $28,49\left(9,94 \mathrm{E}^{-06}\right)$ & $19,82(0,001)$ & $5,86(0,21)$ & & $3,14(0,53)$ \\
\hline$*$
\end{tabular}

Here R: resistant; MR: moderately resistant; MS: moderately susceptible; S: susceptible; HS: highly susceptible.

When different groupings of the reactions were performed, the significance of the two tests were highly significant for SAT2017 and SAT2018, but not for GCH2018 and SAT2019, except for the test of goodness of fit for GCH2018 in case of the grouping (R+MR+MS; S+HS) with P-value of 0.04 (Table 5).

Table $5 \chi 2$ (P-value) for the tests of independence and goodness of fit of the leaf rust reactions at the adult plant stage of FIGS-LR and GCP subsets of barley at Sidi Allal Tazi (SAT2017, SAT2018 and SAT2019) and Guich (GCH2018).

\begin{tabular}{lcccc}
\hline \multirow{2}{*}{ Group of reactions } & SAT2017 & SAT2018 & SAT2019 & GCH2018 \\
\cline { 2 - 4 } & \multicolumn{4}{c}{ Test of independence } \\
\hline R, MR+MS, S+HS & $17.61(0.0001)$ & $11.8(0.003)$ & $0.16(0.92)$ & $3.64(0.162)$ \\
R+MR+MS, S+HS & $16.76(4.25 \mathrm{E}-05)$ & $8.30(0.004)$ & $0.0004(0.98)$ & $3.51(0.061)$ \\
\hline Group of reactions & \multicolumn{4}{c}{ Test of goodness to fit } \\
\hline R, MR+MS, S+HS & $27.82(9.11 \mathrm{E}-07)$ & $18.31(0.0001)$ & $0.23(0.89)$ & $4.66(0.097)$ \\
R+MR+MS, S+HS & $25.28(4.97 \mathrm{E}-07)$ & $14.62(0.0001)$ & $0.001(0.98)$ & $4.44(0.04)$ \\
\hline
\end{tabular}

Here R: resistant; MR: moderately resistant; MS: moderately susceptible; S: sensible; HS: highly susceptible

\section{Predictive modeling of the reaction to leaf rust}

At the seedling stage, the tested machine learning models did not perform similarly for the two LR isolates. For ISO-MRC isolate, all models yielded a significant medium to very high accuracy. The maximum accuracy $(0.94)$ was reached using the BCART model and was then chosen as the best model. The remaining modeling parameters showed the strong mathematical relationship between the reaction to ISO-MRC and the environmental characteristics (Table 6). However, the modeling pattern was opposite for ISO-SAT isolate where all the models were not significantly accurate (Table 7), since the accuracy was similar to the "No Information Rate" and hence 

than sensitivity for all tested models.

408

409

410 isolate ISO-MRC at the seedling stage.

\begin{tabular}{llllll}
\hline Performance Measures & k-NN & SVM & RF & NNET & BCART \\
\hline Accuracy & 0.716 & 0.686 & 0.961 & 0.912 & 0.941 \\
95\% CI & $(0.62-0.80)$ & $(0.59-0.77)$ & $(0.90-0.99)$ & $(0.84-0.96)$ & $(0.88-0.98)$ \\
No Information Rate & 0.5 & 0.5 & 0.5 & 0.5 & 0.5 \\
P-Value [Acc > NIR] & $7.75 \mathrm{E}-06$ & 0.000107 & $8.73 \mathrm{E}-25$ & $4.99 \mathrm{E}-19$ & $2.83 \mathrm{E}-22$ \\
Kappa & 0.431 & 0.373 & 0.922 & 0.824 & 0.882 \\
Sensitivity & 0.549 & 0.529 & 0.922 & 0.824 & 0.882 \\
Specificity & 0.882 & 0.843 & 1 & 1 & 1
\end{tabular}

411 Here k-NN: K-nearest neighbors KNN; SVM: Support Vector Machine; RF: Random Forest; BCART: Bagged 412 Carts

413

414

415

416

Table 7 Performance measures of different machine learning models for resistance of barley accessions to leaf rust isolate ISO-SAT at the seedling stage.

\begin{tabular}{llllll}
\hline Performance Measures & k-NN & SVM & RF & NNET & BCART \\
\hline Accuracy & 0.722 & 0.556 & 0.597 & 0.542 & 0.653 \\
$95 \%$ CI & $(0.60-0.82)$ & $(0.43-0.67)$ & $(0.47-0.71)$ & $(0.42-0.66)$ & $(0.53-0.76)$ \\
No Information Rate & 0.639 & 0.639 & 0.639 & 0.639 & 0.639 \\
P-Value [Acc > NIR] & 0.086782 & 0.943047 & 0.805548 & 0.965561 & 0.455707 \\
Kappa & 0.355 & 0.0368 & 0.089 & -0.0189 & 0.228 \\
Sensitivity & 0.87 & 0.652 & 0.739 & 0.674 & 0.761 \\
Specificity & 0.462 & 0.385 & 0.346 & 0.308 & 0.462 \\
\hline
\end{tabular}

417 Here k-NN: K-nearest neighbors KNN; SVM: Support Vector Machine; RF: Random Forest; BCART: Bagged Carts

For the APR, no model performed significantly for the two locations (Table 8). Accuracy was higher for all models, however, the unbalanced data due to the higher number of resistant genotypes make the model not performing better than the naïve model because of the low values of specificity and high value of "No Information Rate". Among the tested models, RF was the best model for all locations.

424 
Table 8 Performance measures of the best machine learning model (Random Forest) for the prediction of leaf rust adult plant resistance of barley accessions.

\begin{tabular}{llll}
\hline \multirow{2}{*}{ Model } & $(\mathrm{SAT}+\mathrm{GCH})$ & $\mathrm{SAT}$ & $\mathrm{GCH}$ \\
\cline { 2 - 4 } & $\mathrm{RF}$ & $\mathrm{RF}$ & $\mathrm{RF}$ \\
\hline Accuracy & 0.81 & 0.81 & 0.84 \\
$95 \% \mathrm{CI}$ & $(0.741,0.864)$ & $(0.691,0.903)$ & $(0.719,0.918)$ \\
No Information Rate & 0.80 & 0.75 & 0.85 \\
P-Value [Acc > NIR] & 0.47 & 0.15 & 0.72 \\
Kappa & 0.31 & 0.42 & 0.20 \\
Sensitivity & 0.92 & 0.96 & 0.94 \\
Specificity & 0.35 & 0.40 & 0.22 \\
\hline
\end{tabular}

431

432

433

434

435

436

437

438

439

440

441

442

443

444

445

446

447

448

449

450

451

452

453

454

455

456

457

458

459

460

Here SAT: Allal Tazi; GCH: Guich; RF: Random Forest

SAT: Allal Tazi; GCH: Guich; RF: Random Forest

(1) 
pathogen population which lead to the emergence of new races, and eventual breakdown the effectiveness of resistance genes. Several studies reported that the frequency of virulence for the resistance gene Rph4 was increased in Queensland during the 1980s, possibly due to the widespread use of the cultivar Grimmett, which possesses Rph4 (Cotterill et al. 1995; Burdon et al. 2014; Li et al. 2014; Niks et al. 2015). Woldeab et al. (2006) reported the detection of eleven new pathotypes since 1992-2001 in Australia and seven new pathotypes in Ethiopia in 2003 and 2004. Virulence has been detected for most known seedling $R p h$ genes in various barley growing regions throughout the world. In Australia, only Rph3, Rph7, Rph11, Rph14, Rph15, and Rph18 of the characterized major genes were still effective to prevailing pathotypes (Cotterill et al. 1995; Park 2003). However, pathotypes virulent to Rph3 were detected in New Zealand (Cromey and Viljanen-Rollinson 1995), and the virulence for Rph7 has been identified in Israel (Golan et al.1978), Morocco (Parlevliet et al. 1981), and North America (Steffenson et al. 1993). Virulence for Rph11 and Rph14 has also been found frequently in many parts of the world (Fetch et al. 1998), and virulence to Rph15 was reported by Sun et al. (Sun 2007). Therefore, an accession with LR resistance at the seedling stage alone might not provide durable and effective resistance (Singh 1992; Park 2008; Singh et al. 2015).

Only few accessions; IG 143945, IG 144000, IG 144140, IG 144064 from GCP subset, and accessions IG 28613 , IG 28636, IG 33039 from FIGS_LR subset showed resistant (R) to moderately resistant (MR) response at the adult plant stages across all environments. Of the 21 Bowman differential lines tested at the adult plant stage at Sidi Allal Tazi, only one-line displayed moderately resistant reaction to LR field population (unpublished data). Most probably, the LR resistant accessions identified in this study may possess either new $R$ genes or allelic variant of existing $R$ genes or a combination of both. A high-density genotyping and genome wide association studies seem to be logical step to dissect the resistance diversity. These putative $R$ genes could be either pyramided or used sequentially to ensure a better $R$ gene deployment strategy.

Since there are several accessions at the adult plant stage with MR and MS reactions or with slow progression of the disease based on the area under the disease progress curve (data not presented) under heavy rust epidemics, partial resistance and slow rusting mechanism could be considered to ensure a race non-specific and a more durable resistance. Several studies have promoted partial and non-race specific resistance in case of rusts and powdery mildew in barley and wheat as this type of resistance is available in some commercial varieties (Parlevliet and Kuiper 1977; Andres and Wilcoxon 1986; Niks et al. 2000; Stuthman et al. 2007). Several APR genes were well characterized and deployed in wheat to control rust diseases (Park and McIntosh 1994). In barley, three genes governing APR to LR have been identified and used (Rph20, Rph23, and Rph24) (Hickey et al. 2011; Singh et al. 2015; Ziems et al. 2017). Even if there are no reports of virulence for Rph20, Rph23 or Rph24, identifying new APR resistance genes for LR are essential for diversifying resistance and to promote gene pyramiding to increase resistance levels. Marker assisted selection (MAS) provides an opportunity to breeders to pyramid the APR genes in barley.

The Focused Identification of Germplasm Strategy (FIGS) has been promoted by ICARDA genebank as an innovative approach for efficient mining the genebank holdings to better respond to seed requests for specific traits sought by breeders. This approach has shown its efficiency in identifying novel sources of resistance to powdery 
mildew, yellow and stem rusts, Sunn pest, and Russian wheat aphid in wheat (Bhullar et al. 2009; El Bouhssini et al. 2009, 2011; Bari et al. 2012, 2014), and to net blotch of barley (Endresen et al. 2011). This study included the first attempt to compare FIGS with another subset, the Reference set of the Generation Challenge Program (GCP) selected from the global barley core collection based on diversity using SSR markers. The tests of independence showed that the dependence of LR reaction on sub-setting approach varied among the isolates at the seedling stage, and between environments at the adult plant stage. It appears that the dependence is mainly found for ISO-MRC isolate and under heavy infection level experienced at Sidi Allal Tazi. Similarly, the tests of goodness of fit showed that the differences between FIGS-LR and GCP for percentages of accessions with different reactions varied with the isolate at the seedling stage, and on sites and years for the adult plant stage. FIGS sub-setting using filtering approach has allowed to identify higher percentages of accessions with R and MR reactions compared to GCP subset in case of field tests (except SAT2018). Modeling outcomes using machine learning approach were dependent on the isolates, and the environments, and associated pathogen field populations. The reduced sample size as well as the non-balance between the two classes (Resistant and Susceptible) could explain the limitation of the machine learning models. The results showed the need for further fine tuning of FIGS approach to consider the diversity of virulence of the pathogen populations using larger subsets. Since the GCP reference set included a larger number of accessions with $\mathrm{R}$ and MR reactions at both the seedling and the adult plant stages. It will be interesting to compare both sub-setting methods in yielding new different effective genes. This can be investigated using molecular markers or by screening the identified sources of resistance to a larger number of isolates with different virulence spectrums.

\section{Conclusion}

This current study suggests that the trait mining approach can be an efficient alternative to the core collection method. The resistant and moderately resistant accessions at the seedling and the adult plant stages in this study are valuable resources of $P$. hordei resistance and can lead towards effective and durable resistance against $P$. hordei when combined with appropriate gene deployment strategies. The evaluation of larger subsamples in different environments, and against different pathotypes will allow the fine tuning of FIGS sub-setting approach using machine leaning.

Supplemental Table S1. List of 188 accessions from the reference set constructed within the Generation Challenge Program (GCP) used in the study and relevant information such as IG \#, Origin, and collection site. Supplemental Table S2. List of 86 barley accessions selected using filtering approach of the Focused Identification of Germplasm Strategy (FIGS_LR) and relevant information such as IG \#, Origin, and collection site. 


\section{Declarations}

541 Acknowledgements: The authors would like to thank Dr. Kenneth Street for his help in developing FIGS subset

542 and Mr. Amer El-Omrani, the technician at Sidi Allal Tazi experimental station for his help with field

543 experiments.

544 Funding: This study is supported financially through GIZ-attributed funding to ICARDA genebank and the

545 CAIGE-GRDC-ICA00010 project.

546 Data availability statement

547 The data that support the findings of this study are available in the ICARDA genebank database and can be

548 obtained upon request from the corresponding author.

549 Compliance with ethical standards

550 This work does not contain any studies with human participants or animals performed by any of the authors

\section{Conflict of interest}

552 The writers declare that they have no known conflicting financial interests or personal relations that may have had an impact on the work presented in this article.

555 
Andres MW, Roy DWilcoxson (1986) Selection of Barley for Slow Rusting Resistance to Leaf Rust in

of

Different

Severity.

Crop

Sci

26:

$511-514$. https://doi.org/10.2135/cropsci1986.0011183X002600030015x

561

562

563

564

565

566

567

568

569

570

571

572

573

574

575

576

577

578

579

580

581

582

583

584

585

586

587

588

589

590

591

592

593

594

595

Arnst BJ, Martens JW, Wright GM, Burnett PA, Sanderson FR (1979) Incidence, importance and virulence of Puccinia hordei on barley in New Zealand. Ann Appl Biol 92:185-90

Bari A, Street K, Mackay M, Endresen DTF, De Pauw E (2012) Focused identification of germplasm strategy (FIGS) detects wheat stem rust resistance linked to environmental variables. Genet Resour Crop Evol 59: 14651481.

Bari A, Amri A, Street K, Mackay M, De Pauw E, Sanders R, Nazari K, Humeid B, Konopka J, Alo F (2014) Predicting resistance to stripe (yellow) rust (Puccinia striiformis) in wheat genetic resources using focused identification of germplasm strategy. The Journal of Agricultural Science 152(06):906-916. DOI: $10.1017 / \mathrm{S} 0021859613000543$

Bhullar NK, Street K, Mackay M, Yahiaoui N, Keller B (2009) Unlocking wheat genetic resources for the molecular identification of previously undescribed functional alleles at the Pm3 resistance locus. Proc Natl Acad Sci USA 106: 9519-9524.

Breiman L (2001) Random forests. Machine Learning 45(1):5-32

Brunner S, Keller B, Feuillet C (2000) Molecular mapping of the Rph7.g leaf rust resistance gene in barley (Hordeum vulgare L.). Theor Appl Genet 101:783-788

Burdon JJ, BarrettLG, RebetzkeG, Thrall PH (2014) Guiding deployment of resistance in cereals using evolutionary principles. Evol Appl 7:609-624. 10.1111/eva.12175

Chicaiza O, Franckowiak JD, and Steffenson BJ (1996) New sources of resistance to leaf rust in barley. In: Slinkard AE, Scoles GJ, Rossnagel BG (eds) Proc Fifth Int Oat Conf \& Seventh Int Barley Genet Symp. Saskatoon, pp 706-708.

CGIAR Generation Challenge Programme (2012) Paper No 3: Genetic stocks). Position Papers: GCP's research component. Texcoco, Mexico. 22 pp.

Clifford BC (1985) Barley leaf rust. In: Roelfs AP, Bushnell WR (eds) The cereal Rusts. Diseases, Distribution, Epidemiology and Control, Vol II. Harcourt Brace Jovanovich, Publishers Orlando, Florida 32887, pp 173-205

Cotterill PJ, Rees RG, Platz GJ, Dill-Macky R (1992) Effects of leaf rust on selected Australian barleys. Aus J Exp Agric 32: 747-751

Cotterill PJ, Park RF, ReesRG (1995) Pathogenic specialization of Puccinia hordei Otth. in Australia, 19661990. Aus J Agric Res 46: 127-134

Cromey MG, Viljanen-Rollinson SLH (1995) Virulence of Puccinia hordei on barley in New Zealand from 1990 to 1993. N Z J Crop Hortic Sci 23:115-19

Derevnina L, Singh D, Park RF (2013) Identification and characterization of seedling and adult plant resistance to Puccinia hordei in Chinese barley germplasm. Plant Breeding 132(6): 571-579. doi:10.1111/pbr.12082

De Pauw, Oweis T, Youssef J (2008) Integrating expert knowledge in GIS to locate biophysical potential for water harvesting: Methodology and a case study for Syria. ICARDA, Aleppo, Syria pp 59 
Dwivedi SL, Stalker HT, Blair MW, Bertioli D, Upadhyaya HD, Nielen S et al. (2008) Enhancing crop gene pool with beneficial traits using wild relatives. Plant Breed 30:179-230. 10.1002/9780470380130.

El Bouhssini M, Street K, Joubi A, Ibrahim Z, Rihawi F (2009) Sources of wheat resistance to sunn pest, Eurygaster integriceps Puton, in Syria. Genet Resour Crop Evol 56: 1065-1069

El Bouhssini M, Street K, Amri A, Mackay M, Ogbonnaya FC et al (2011) Sources of resistance in bread wheat to Russian wheat aphid (Diuraphis noxia) in Syria identified using the Focused Identification of Germplasm Strategy (FIGS). Plant Breed 130: 96-97

Elmansour H, Singh D, Dracatos PM, Park RF (2017). Identification and characterization of seedling and adult plant resistance to Puccinia hordei in selected African barley germplasm. Springer Science+Business Media Dordrecht. DOI 10.1007/s10681-017-1902-8

Endresen DTF, Street K, Mackay M, Bari A, De Pauw E (2011) Predictive association between biotic stress traits and ecogeographic data for wheat and barley landraces. Crop Sci 51: 2036-2055

Endresen DTF, Street K, Mackay M, Bari A, Amri A, De Pauw E, Nazari K, Yahyaoui A, (2012) Sources of resistance to stem rust (ug99) in bread wheat and durum wheat identified using focused identification of germplasm strategy (FIGS). Crop Science 52: 764-773

FAO. FAOStat, http://www.fao.org/faostat/en/\#rankings/countries_by_commodity (2017)

Fetch TG, Steffenson BJ, Jin Y (1998) Worldwide virulence of Puccinia hordei on barley. Phytopathology 88:S28

Fick SE and Hijmans RJ (2017) WorldClim 2: new 1km spatial resolution climate surfaces for global land areas. International Journal of Climatology 37 (12): 4302-4315

Figueroa M, Upadhyaya NM, Sperschneider J, Park RF, Szabo LJ, Steffenson B, Dodds PN (2016) Changing the Game: Using Integrative Genomics to Probe Virulence Mechanisms of the Stem Rust Pathogen Puccinia graminis f. sp. tritici. Frontiers in Plant Science 1-10. http://doi.org/10.3389/fpls.2016.00205

Frankel OH, Brown AHD (1984) Current plant genetic resources- a critical appraisal. In: Chopra VL, Joshi BC, Sharma RP, and Bansal HC (eds) Genetics: New Frontiers. ( Oxford \& IBH Publ Co, New Delhi, India, pp 113

Global Crop Diversity Trust (2008) Global strategy for the ex situ conservation and use of barley germplasm [online]. Available from http://www.croptrust.org/documents/web/ BarleyStrategy FINAL 27Oct08.pdf

Golan T, Anikster Y, Moseman JG, Wahl I (1978) A new virulent strain of Puccinia hordei. Euphytica 27:18589

Golegaonkar, PG, Singh D, Park RF (2009) Evaluation of seedling and adult plant resistance to Puccinia hordei in barley. Euphytica 166: 183-197

Gollin D, Smale M, Skovmand B (2000) Searching an ex situ collection of wheat genetic resources. Am J Agric Econ 82: 812-827

Gollin, D., M. Smale, and Skovmand B (2000) Searching an ex situ collection of wheat genetic resources. American Journal of Agricultural Economics 82(4):812-827. https://doi.org/10.1111/0002-9092.00083

Griffey CA, Das MK, Baldwin RE, Waldenmaier CM (1994) Yield losses in winter barley resulting from a new race of Puccinia hordei in North America. Plant Dis 78:256-260

Hajjar R, and Hodgkin T (2007) The use of wild relatives in crop improvement: A survey of developments over the last 20 years. Euphytica 156:1-13. doi:10.1007/s10681-007-9363-0 
Hickey LT, Lawson W, Platz GJ, Dieters M, German S, et al. 2011. Mapping Rph20: a gene conferring adult plant resistance to Puccinia hordei in barley. Theor Appl Genet 123:44-68

Hintum Th van, Menting F (2003) Diversity in ex situ genebank collections of barley. In: Bothmer R von, Hintum Th van, Knüpffer H and Sato K (eds) diversity in Barley (Hordeum vulgare). Elsevier Science BV, Amsterdam, The Netherlandspp 247-257

Hsu CW, Chang CC, Lin CJ (2010) A Practical Guide to Support Vector Classification, Department of Computer Science, National Taiwan University, Taipei, Taiwan

ICARDA (2013) A new approach to mining Agricultural gene banks - to Speed the pace of research Innovation for food security. "FIGS" - the Focused Identification of Germplasm Strategy. Research in Action 3. International Center for Agriculture Research in Dry Areas, Beirut, Lebanon.

ICARDA (2015) ICARDA Annual Report 2014. International Center for Agricultural Research in the Dry Areas, Beirut, Lebanon. 56 pp. ISSN: 92-9127-290-6

Kavanagh PJ, Singh D, Bansal UK, and Park RF (2017) Inheritance and characterization of the new and rare gene Rph25 conferring seedling resistance in Hordeum vulgare against Puccinia hordei. Plant Breed 136:908-912

Khazaei H, Street K, Bari A, Mackay M, Stoddard FL (2013) The FIGS (Focused Identification of Germplasm Strategy) Approach Identifies Traits Related to Drought Adaptation in Vicia faba Genetic Resources. PLoS ONE 8(5): e63107. https://doi.org/10.1371/journal.pone.0063107

Knüpffer H, Hintum Th van (1995) The barley core collection: an international effort. In: (T. Hodgkin T, Brown AHD, Hintum Th van and Morales EAV (eds) Core Collections of Plant Genetic Resources John Wiley and Sons, Chichester, UK. pp. 171-178

Kołcz A (2000) N-tuple Network, CART, and Bagging, in Neural Computation,

Kotsiantis SB (2007) Supervised machine learning: a review of classification techniques, Informatica

Kuhn M (2008) Building Predictive Models in R Using the caret Package, Journal of Statistical Software 28: 126

Li LG, Cai L, Zhang XX, Zhang T (2014) Potentially novel copper resistance genes in copper-enriched activated sludge revealed by metagenomic analysis. Appl Microbiol Biotechnol 98: 10255-10266.

Mackay M (1990) Strategic planning for effective evaluation of plant germplasm. In: Srivastava JP, Damania AB (eds) Wheat genetic resources: meeting diverse needs. John Wiley \& Sons, Chichester, pp 21-25

Mackay M (1995) One core collection or many? In: Hodgkin T, Brown AHD, van Hintum TJL, Morales EAV (eds) Core collections of plant genetic resources. John Wiley \& Sons Ltd, Chichester, pp 199-210

Mackay M, Street K (2004) Focused identification of germplasm strategy - FIGS. In: Black CK, Panozzo JF, Rebetzke GJ (eds) Proceedings of the 54th Australian Cereal Chemistry Conference and the $11^{\text {th }}$ Wheat Breeders' Assembly, pp 138-141. Royal Australian Chemical Institute (RACI), MelbourneMcIntosh R (1988) The Role of Specific Genes in Breeding for Durable Stem Rust Resistance in Wheat and Triticale. In: Simmonds S, Rajaram NW (eds)Breeding strategies for resistance to rust of wheat pp 1-9. Mexico, DF: CIMMYT.

Niks RE, Walther U, Jaiser H, Martínez F, Rubiales D, Andersen O, Flath K, Gymer P, Heinrichs F, Jonsson R, Kuntze L, Rasmussen M, Richter E (2000) Resistance against barley leaf rust (Puccinia hordei) in WestEuropean spring barley germplasm. Ann appL Bid 92: 185-190 
Niks RE, Qi XQ, Marcel TC (2015) Quantitative resistance to biotrophic filamentous plant pathogens: concepts, misconceptions and mechanisms. in: vanAlfen NK (ed) Annual Review of Phytopathology pp 445-470

Park RF, McIntosh RA, (1994) Adult plant resistances to Puccinia recondita f. sp. tritici in wheat. N Z J Crop Hortic Sci 22:151-158

Park RF (2003) Pathogenic specialization and pathotype distribution of Puccinia hordei in Australia, 1992 to 2001. Plant Dis 87:1311-16

Park RF, (2008) Breeding cereals for rust resistance in Australia. Plant Pathology 57: 591-602. 10.1111/j.13653059.2008.01836.x

Park RF, Golegaonkar P, Derevnina L, Sandhu K, Karaoglu H, Elmansour H, Dracatos P, Singh D (2015) Leaf Rust of Cultivated Barley: Pathology and Control. Annual Review of Phytopathology 53: 565-589

Parlevliet JE, Kuiper HJ (1977) Partial resistance of barley to leaf rust, Puccinia hordei. IV. Effect of cultivar and development stage on infection frequency. Euphytica 26:249-255.

Parlevliet JE, Van Der Beek JG, Pieters R (1981) Presence in Morocco of brown rust, Puccinia hordei, with a wide range of virulence to barley. Cereal Rusts Bull 9:3-8

Peterson RF, Campbell AB,Hannah AE (1948) A diagrammatic scale for estimating rust intensity of leaves and stem of cereals. Can J Res 26: 496-500

Qualset C (1975) Sampling germplasm in a center of diversity: an example of disease resistance in Ethiopian barley. Crop genetic resources for today and tomorrow 81-96

R Core Team (2018) R: A language and environment for statistical computing. R foundation for statistical computing, Vienna, Austria, https://www.R-project.org/

Rehman S, Amouzoune M, Hiddar H, Aberkane H, Benkirane R, Filali-Maltouf A, Al-Jaboobi M, Acqbouch L, Tsivelikas A, Verma R, Kehel Z, \& Birouk A, \& Amri A (2020) Traits discovery in Hordeum vulgare sbsp. spontaneum accessions and in lines derived from interspecific crosses with wild Hordeum species for enhancing barley breeding efforts. Crop Sci 1-15. DOI: 10.1002/csc2.20360

Russell JR, Baum M, Ceccarelli S, Close TM, Grando S, Hayes PM, Matus I, Marshall DF, Del Poza A, von Korff Schmising M, Waugh R (2008) Genomic dissection of tolerance to drought stress in wild barley. $10^{\text {th }}$ International Barley Genetics Symposium, Alexandria, Egypt, 5-10 April 2008

Sandhu KS, Singh D, Park RF (2014) Characterising seedling and adult plant resistance to Puccinia hordei in Hordeum vulgare. Ann Appl Biol 165:117-129

Singh R (1992) Genetic association of leaf rust resistance gene Lr34 with adult plant resistance to stripe rust in bread wheat. Phytopathology 82:835-838

Singh D, Dracatos P, Derevnina L, Zhou MX, Park RF (2015) Rph23: A new designated additive adult plant resistance gene to leaf rust in barley on chromosome 7H. Plant Breed. 134:62-69

Stakman EC, Stewart DM, Loegering WQ (1962) Identification of physiologic races of Puccinia graminis var. tritici. USDA Agric Res Serv E617:5-53

Steffenson BJ, Jin Y, Griffey CA (1993) Pathotypes of Puccinia hordei with virulence for the barley leaf rust resistance gene Rph7 in the United States. Plant Dis. 77:867-69

Street K, Mackay M, Zuev E, Kaur N, El Bouhssini M, Konopka J, Mitrofanova O (2008) Diving into the genepool: a rational system to access specific traits from large germplasm collections. In: ( Appels R, Eastwood R, 
Lagudah E, Langridge P, Mackay M, Mcintyre L, Sharp P(eds) Proceedings of the $11^{\text {th }}$ International Wheat GeneticsSymposium , pp 28-31. Brisbane, Australia: Sydney University Press

Stubbs RW, Prescot JM, Saari EE, Dubi HJ (1986) Cereal Disease Methodology Manual. Centro Internacional de Mejoramiento de Maizey Trigo (CIMMYT), Mexico, Pages: 46

Stuthman DD, KJ, Leonard J, Miller-Garvin (2007) Breeding Crops for Durable Resistance to Disease. Advances in Agronomy, Academic Press 95: 319-367

Sun Y (2007) Study of Puccinia hordei and its host resistances in Hordeum vulgare. PhD Thesis, North Dakota State Univ, Fargo, ND

Upadhyaya HD, Oritz R. (2001) A mini core subset for capturing diversity and promoting utilization of chickpea genetic resources in crop improvement. Theor Appl Genet 102:1292-1298

USDA United States Department of Agriculture Foreign Agricultural Service (FAS), Ministry of Agriculture, Rabat. (2018). (https://gain.fas.usda.gov/RecentGAIN/ Publications/Grainand/Feed/AnnualRabatMorocco_331-2018.pdf)

Venables WN, Ripley BD (2002) Modern applied statistics with S, 4th edn. Springer, New York

Woldeab G, Fininsa C, Singh H, Yuen J (2006) Virulence spectrum of Puccinia hordei in barley production systems in Ethiopia. Plant pathology 55(3):351-357

Xu Y (2010) Plant genetic resources: Management, evaluation and enhancement. In: Molecular plant breeding. Wallingford, UK, CAB International, 15-194

Yu X, Kong HY, Meiyalaghan V, Casonato S, Chng S, Jones EE, Butler RC, Pickering R, Johnston PA (2018) Genetic mapping of a barley leaf rust resistance gene Rph26 introgressed from Hordeum bulbosum. Theor Appl Genet 131:2567-2580. https ://doi.org/10.1007/s0012 2-018-3173-8

Zadoks JC, Chang TT, Konzak CF (1974) A decimal code for the growth stages of cereals. Weed Res 14: 415-421 
Figures
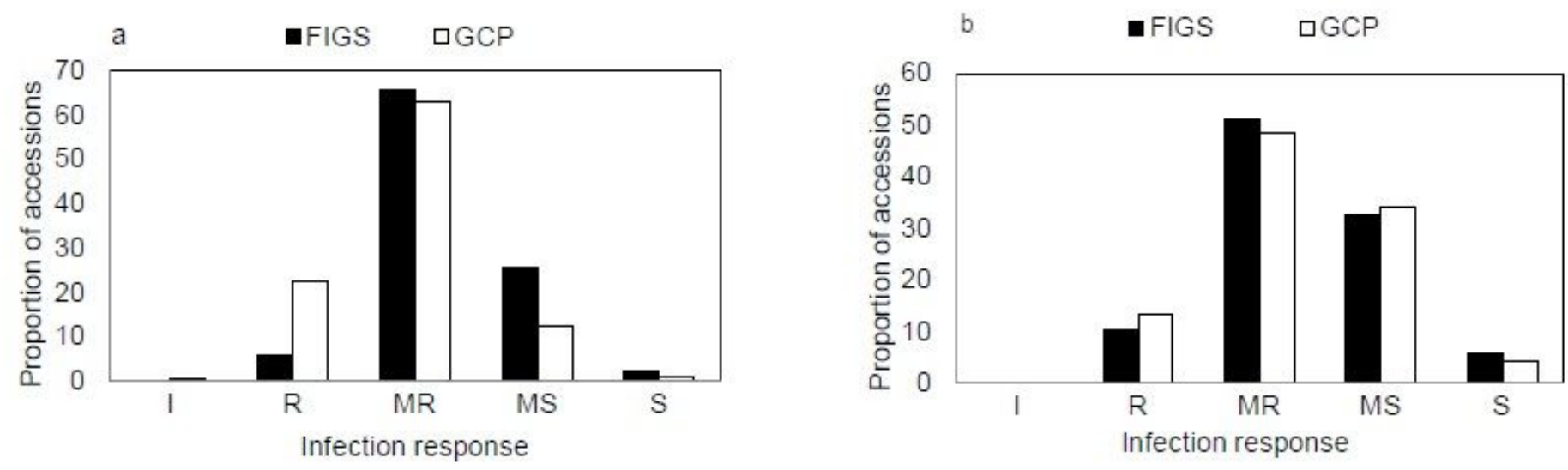

Figure 1

Frequency distributions of LR disease reactions of barley FIGS_LR and GCP subsets evaluated at the seedling stage against ISO-MRC (a) and ISO-SAT isolates (b). Here I = immune, R = resistant, MR = moderately resistant, $\mathrm{MS}=$ moderately susceptible, and $\mathrm{S}=$ susceptible.
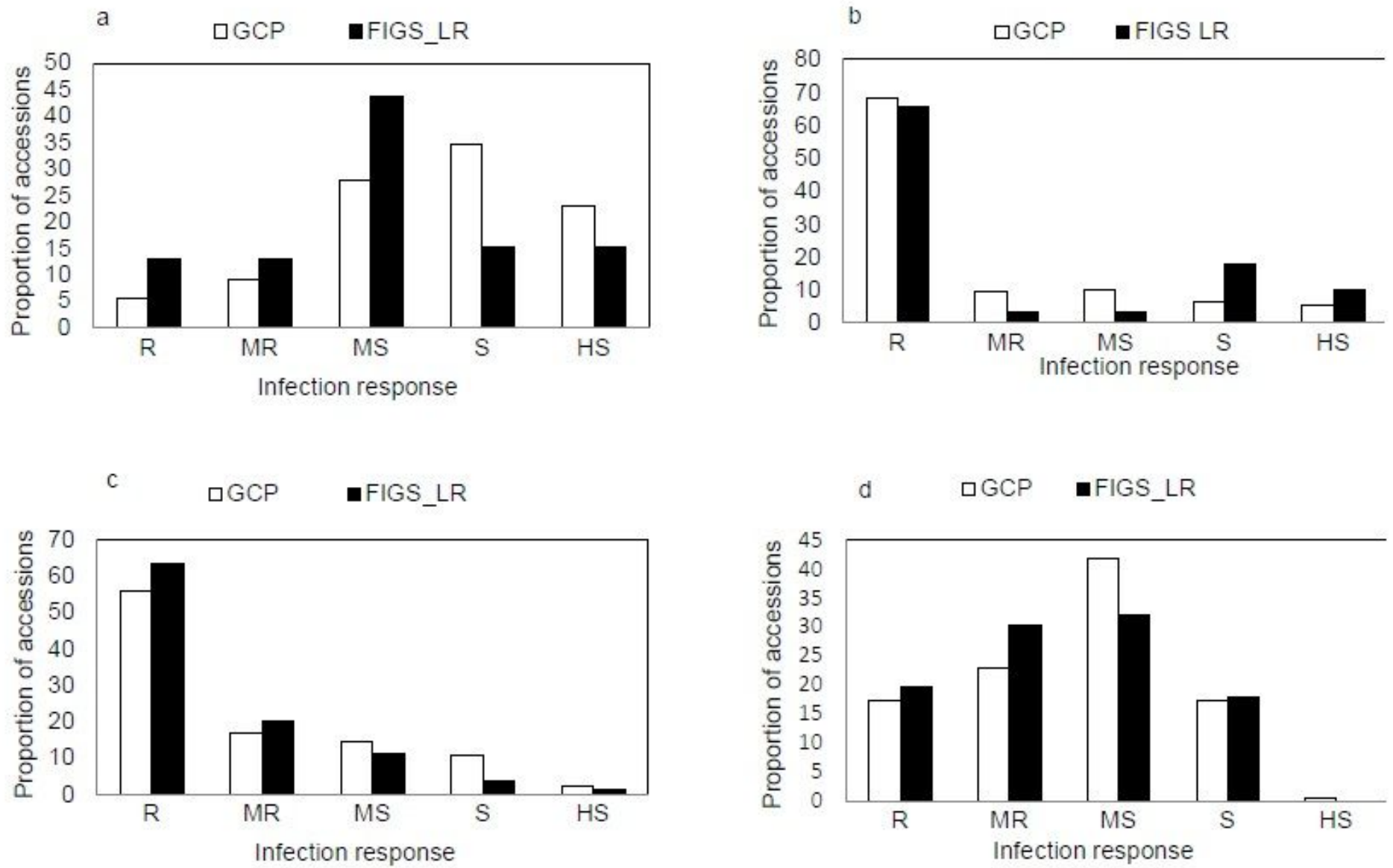

Figure 2 
Frequency distributions of the adult plant resistance (APR) of FIGS_LR and GCP subsets to leaf rust under field conditions at Sidi Allal Tazi during (a) 2017 (SAT2017), (b) 2018 (SAT2018), (d) 2019 (SAT2019) cropping seasons, and at Guich during 2018 (GCH2018 (c)). Here R = resistant, MR= moderately resistant, $\mathrm{MS}=$ moderately susceptible, and $\mathrm{S}=$ susceptible.

\section{Supplementary Files}

This is a list of supplementary files associated with this preprint. Click to download.

- SupplementaryTableS1.xlsx

- SupplementaryTableS2.xlsx 